\section{Review author's response: pitfalls in isolating lipid rafts}

The letter by Nothdurfter et al. regarding the methodological pitfalls associated with isolating lipid raft membranes is a welcome discussion about a topic that has evoked considerable debate in this field. We thank the authors for voicing their insightful comments and for providing experimental observations and suggestions to overcome some of the technical problems facing those investigating membrane microdomains. Isolation of detergent-resistant membranes remains a near-universal approach for obtaining lipid raft/caveolae membranes; however, it is important to emphasize that this experimental manipulation results in biochemical and operationally defined membrane microdomains, rather than iconic structures. It is worth reiterating that alternative fractionation techniques, which do not rely on detergent solubility, have addressed some of these problems ${ }^{1}$, and that these are preferred by some investigators. Both detergent and non-detergent membrane isolations result in defined membrane domains that are probably consistent for a given technique and tissue type. Thus, it is incumbent upon investigators to repeat their own methods exactly from experiment to experiment, and importantly, to clearly explain in their published work complete details of the method so that others can faithfully repeat the results. This should include outlining the details of concentration of detergent, volumes used and the protein-to-detergent ratios. Note, for example, that in Figure 1, Nothdurfter et al. add an internal standard and suggest that tissue types might be a reason for variability in lipid raft/caveloae determinations. However, it would be most informative to see these tissue types compared in experiments using the same detergent conditions and the same density matrix (for example, both using 1\% TX-100 and both separated using discontinuous sucrose gradients). As we suggest in our review article ${ }^{2}$, studies involving the intricacies of signal transduction and lipid rafts should use several complementary approaches, and whenever possible, should use techniques that can be tested in intact cells ${ }^{3}$.

Regarding rafts and neuropsychopharmacology, as has been demonstrated, only a subset of psychotropic drugs associate with rafts ${ }^{4}$. Certain psychoactive compounds such as lithium, which is a simple salt, would not be predicted to be raft associated. It would be useful to know whether raft localization of a number of lipophilic drugs was simply a function of lipid solubility or, as we suspect, a more complex process related to the unique chemical properties of these compounds. We have shown that chemically diverse antidepressants decrease the association of the heterotrimeric $\mathrm{G}$ protein $\mathrm{G} \alpha_{\mathrm{s}}$ with lipid rafts, without altering the raft localization of other signalling proteins or raft marker proteins ${ }^{5}$. This effect, which shows an absolute requirement for chronic drug treatment, still lacks a parsimonious definition, but cannot be explained simply by the association of these drugs with the lipid components of raft domains.

It has been known for some time that modifying membrane lipids could alter cyclic AMP (cAMP) signalling by interfering with or potentiating interactions between $\mathrm{G} \alpha_{\mathrm{s}}$ and adenylyl cyclase ${ }^{6}$. We have previously suggested that association of $\mathrm{G} \alpha_{\mathrm{s}}$ with lipid rafts removes it from membrane signalling cascades, thereby attenuating $\beta$-adrenergic-G $\alpha_{s}-\mathrm{cAMP}$ signalling ${ }^{7}$. By contrast, rafts are obligatory components for signalling through other G-protein cascades such as $5-\mathrm{HT}_{2 \mathrm{~A}} / \mathrm{G}_{\mathrm{q}} / \mathrm{Ca}^{2+}$ (REF. 8.). Thus, as outlined in our Review, lipid rafts modify neurotransmitter signalling through multiple mechanisms and at multiple levels within signalling cascades. The elucidation of these complex mechanisms will occupy considerable future intellectual and experimental energy, and should reveal important new insights for cellular signalling and neuroscience.

John A. Allen* and Mark M. Rasenick*,

\section{Author affiliations}

* Departments of Physiology \& Biophysics and ${ }^{\ddagger}$ Psychiatry, and the Graduate Program in Neuroscience, University of Illinois at Chicago, College of Medicine, Illinois, USA. Correspondence to M.M.R.e-mail: raz@uic.edu

\section{Refences}

Song, K. S. et al. Co-purification and direct interaction of Ras with caveolin, an integral membrane protein of caveolae microdomains. Detergent-free purification of caveolae microdomains. J. Biol. Chem. 271, 9690-9697 (1996)

2. Allen, J. A., Halverson-Tamboli, R. A. \& Rasenick, M. M. Lipid raft microdomains and neurotransmitter signalling. Nature Rev. Neurosci. 8, 128-140 (2007).

3. Lagerholm, B. C., Weinreb, G. E., Jacobson, K. \& Thompson, N. L. Detecting microdomains in intact cell membranes. Annu. Rev. Phys. Chem. 56, 309-336 (2005) Eisensamer, B. et al. Antidepressants and antipsychotic drugs colocalize with 5-HT3 receptors in raft-like domains. J. Neurosci. 25, 10198-10206 (2005).

5. Donati, R. J. \& Rasenick, M. M. Chronic antidepressant treatment prevents accumulation of $\mathrm{G} \alpha$ in cholesterol-rich, cytoskeletal-associated, plasma membrane domains (lipid rafts). Neuropsychopharmacology 30, 1238-1245 (2005).

6. Rasenick, M. M., Stein, P. J. \& Bitensky, M. W. The regulatory subunit of adenylate cyclase interacts with cytoskeletal components. Nature 294, 560-562 (1981).

7. Allen, J. A., Yu, J. Z., Donati, R. J. \& Rasenick, M. M. $\beta$-adrenergic receptor stimulation promotes $\mathrm{G} \alpha_{\mathrm{s}}$ internalization through lipid rafts: a study in living cells. Mol. Pharmacol. 67, 1493-1504 (2005)

8. Bhatnagar, A., Sheffler, D. J., Kroeze, W. K., Compton-Toth, B. \& Roth, B. L. Caveolin-1 interacts with 5- $\mathrm{HT}_{2}$ serotonin receptors and profoundly modulates the signaling of selected $\mathrm{G} \alpha_{q}$-coupled protein receptors. J. Biol. Chem. 279, 34614-34623 (2004). 